Multimedia Appendix 1. The coded taxonomy.

\title{
Clinical module
}

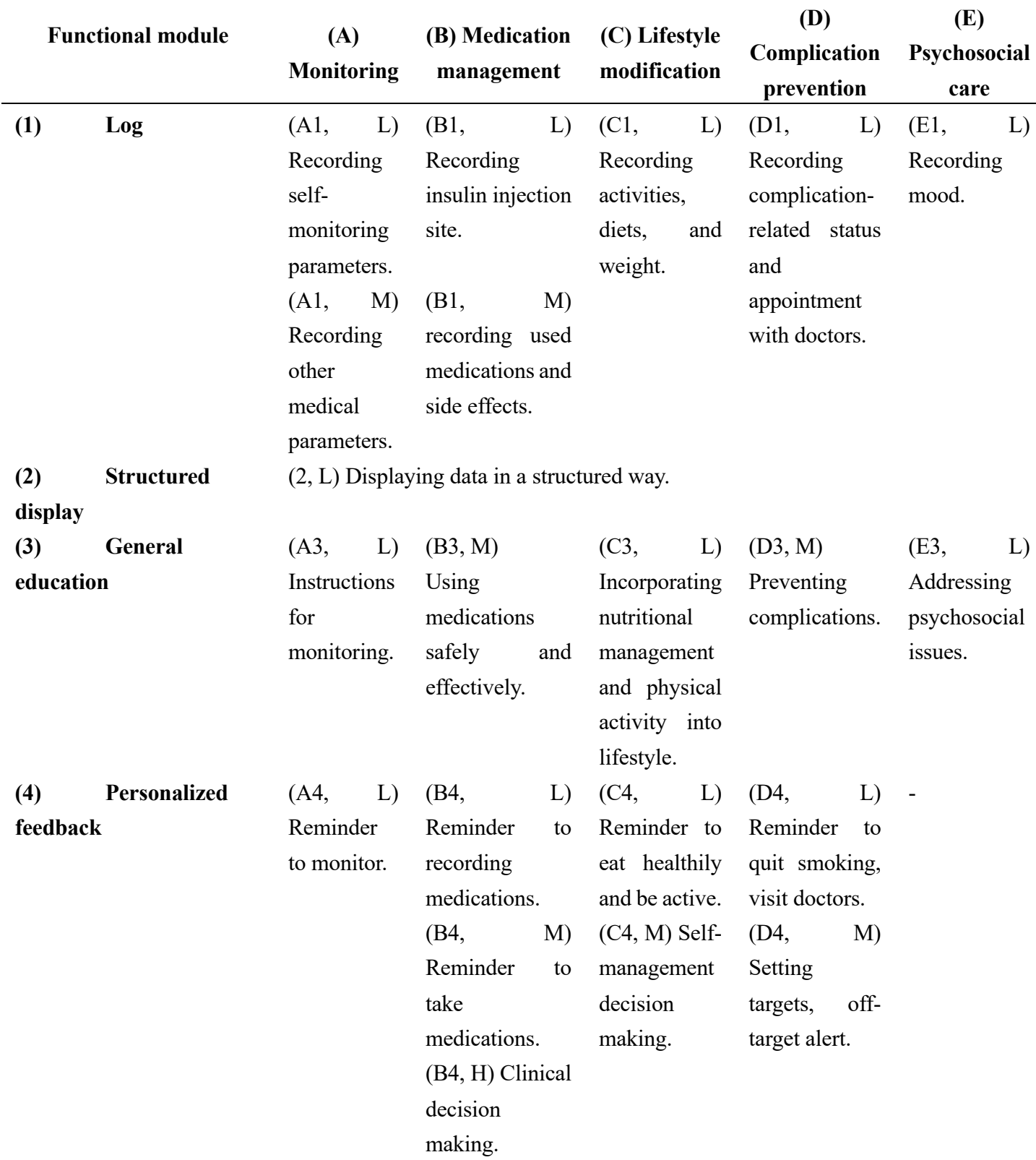

(5) Communication (5, L) General communication, connecting users with their peers and families through social networking, chat forums, or websites.

(5, M) Patient-clinician communication, in-app access to health care providers support or consultation.

a. The risk was coded as L, M and H. L- low risk, M-mediate risk, H-high risk.

b. Clinical module was coded as A-E; functional module was coded as 1-5; the crossing functions by clinical module and functional module were coded like A1, A2. 